\title{
Macrodystrophia lipomatosa - MR imaging of a rare congenital anomaly: review of 3 cases
}

Pallavi Aga, MD
Umesh C Parashari, MD
Anit Parihar, MD
Ragini Singh, MD
Neera Kohli, MD
Rajesh Yadav, MD
Chattrapati Shahuji Maharaj Medical University, Lucknow, Uttar Pradesh,
India

\section{Introduction}

Macrodystrophia lipomatosa (MDL) results from progressive overgrowth of all the mesenchymal elements, with a disproportionate increase in fibro-adipose tissue. This rare congenital abnormality occurs most frequently in the distribution of the median nerve in the upper, and medial plantar nerve in the lower, extremity.'

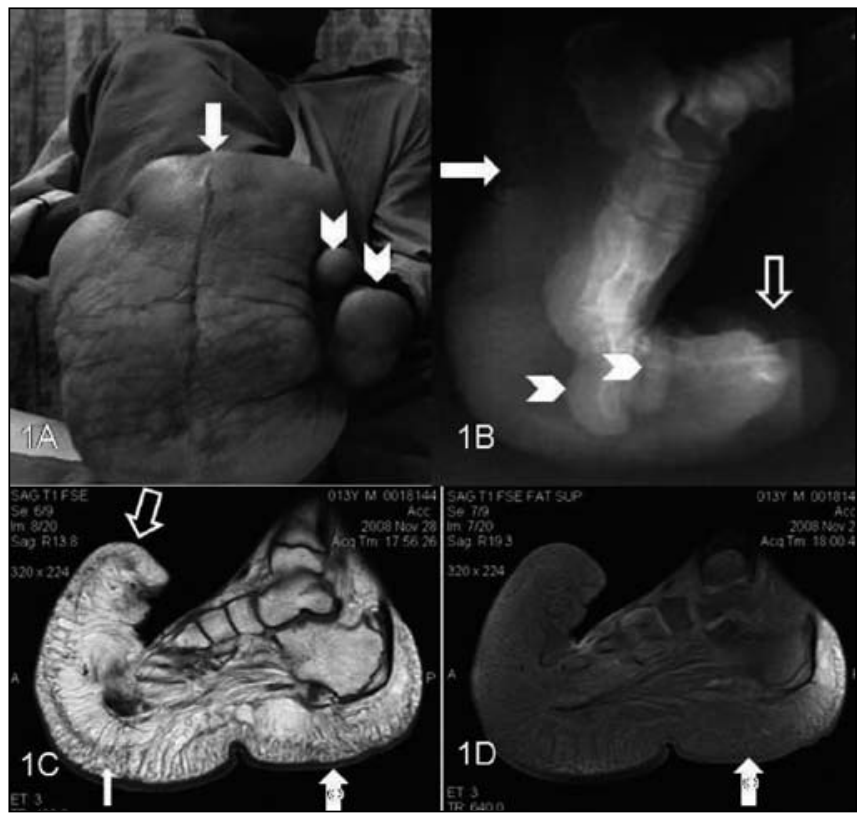

Fig. 1A. Photograph showing swelling on the plantar aspect of the right foot (white arrow). First and 2nd digits are spared (white arrow heads).

Fig. 1B. Plain skiagram of right foot revealing hyperextension of the 3rd, 4th and 5th metatarsophalangeal joints (open white arrow), with overgrowth of distal phalanges. First and 2nd digits are seen normally (white arrow heads). Fig. 1C. T1W sagittal image of right foot revealing diffused thickening of soft tissue (thick white arrow) appearing heterogeneously hyperintense with multiple thin linear low-signal intensity areas consistent with fibrous strands ( thin white arrow). Hyperextension is seen at metatarsophalangeal joints (open white arrow).

Fig. 1D. T1W fat-suppressed sagittal image of right foot shows suppression of the signal from the unencapsulated fat (white arrow).

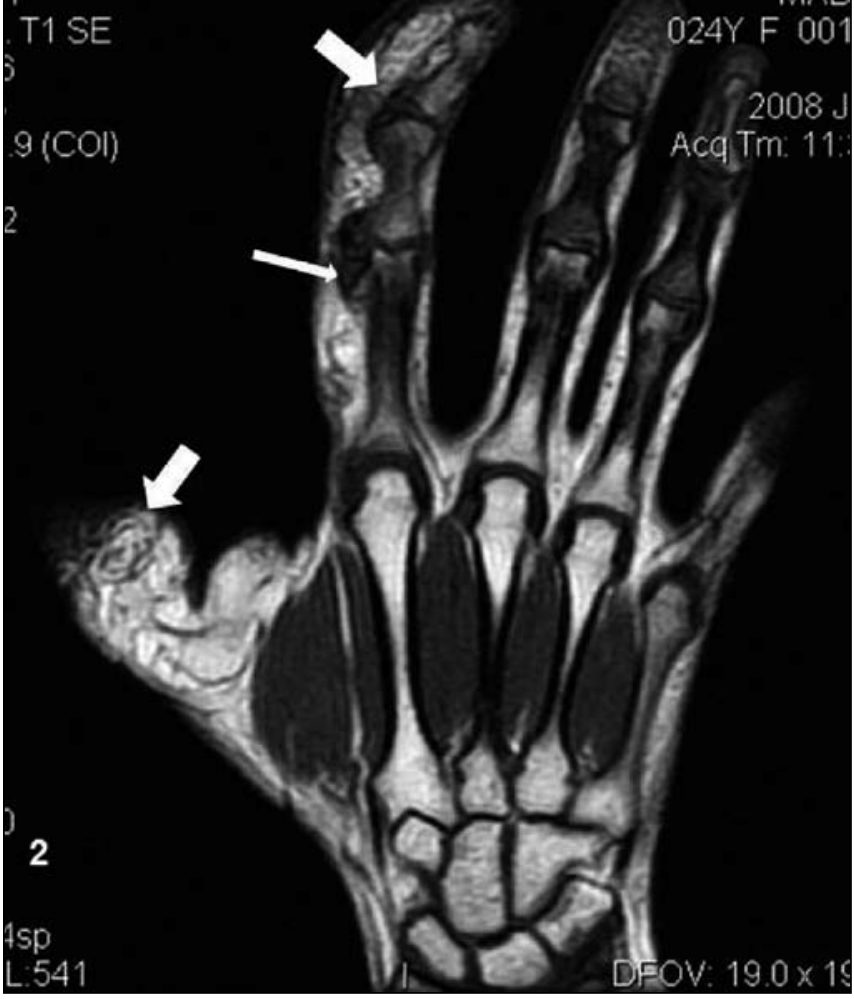

Fig. 2. T1W coronal image of the left hand showing focal hypertrophy of the thumb and index finger (distribution of median nerve) owing to enlarged soft tissue showing a striated pattern with fatty infiltration (thick white arrow). Bony excrescences are seen in involved phalanges (thin white arrow).

Excess of unencapsulated fibro-adipose tissue on magnetic resonance imaging (MRI) is virtually diagnostic and can differentiate this entity from other diseases with similar presentations. Involvement of the ulnar aspect of the hand and the lateral aspect of the foot as seen in our cases is exceedingly rare.

\section{Case report}

Case 1

A 13-year-old boy presented to us from the Department of Plastic Surgery for X-ray of his right foot for a painless soft-tissue mass present since birth (Fig. 1A). Local examination revealed soft, non-oedematous, non-tender, non-pulsatile swelling on the plantar aspect of the right foot. There was no history of fever or trauma or familial gigantism. No bruit or skin changes were present over the mass. The rest of the examination was normal.

On plain skiagram, hyperextension of the 3rd, 4th and 5th metatarsophalangeal joints was found, with overgrowth of distal phalanges. There was no evidence of osteolytic/osteosclerotic changes, periosteal reaction or abnormal calcifications. The rest of the bones 


\section{CASE REPORT}

appeared normal (Fig. 1B). A grey-scale ultrasound with colour Doppler study was done to exclude vascular pathology. Ultrasound revealed a soft-tissue mass with abnormal subcutaneous fat, without dilated vascular channels or calcification.

MR imaging on $1.5 \mathrm{~T}$ units revealed diffused thickening of soft tissue of the right foot, involving the 3rd, 4th and 5th digits, appearing heterogeneously hyperintense on T1- and T2-weighted sequences with multiple thin linear low-signal intensity areas consistent with fibrous strands (Fig. 1C). Fat-suppressed images showed suppression of the soft-tissue areas consistent with unencapsulated fat (Fig. 1D). Areas of hyperintensity were seen in the tarsal bones on T2-weighted fatsuppression images. The rest of the underlying bones revealed normal signal intensity with intact periosteum. Underlying metatarsals appeared to be widened. There was no associated nerve hypertrophy or soft-tissue calcification. No evidence of haemangioma or lymphangioma was seen.

From the above findings, a radiological diagnosis of MDL was made, which was consistent with the histopathology.

\section{Case 2}

A 24-year-old woman presented to our department for MR imaging of painless non-tender and non-pitting swelling in the thumb and index finger of her left hand. The swelling was atraumatic, had been present for a long time, and was slowly increasing in size. There was no history of swelling in any other part of body, and no family history. Overlying skin was normal, and there was no bruit.

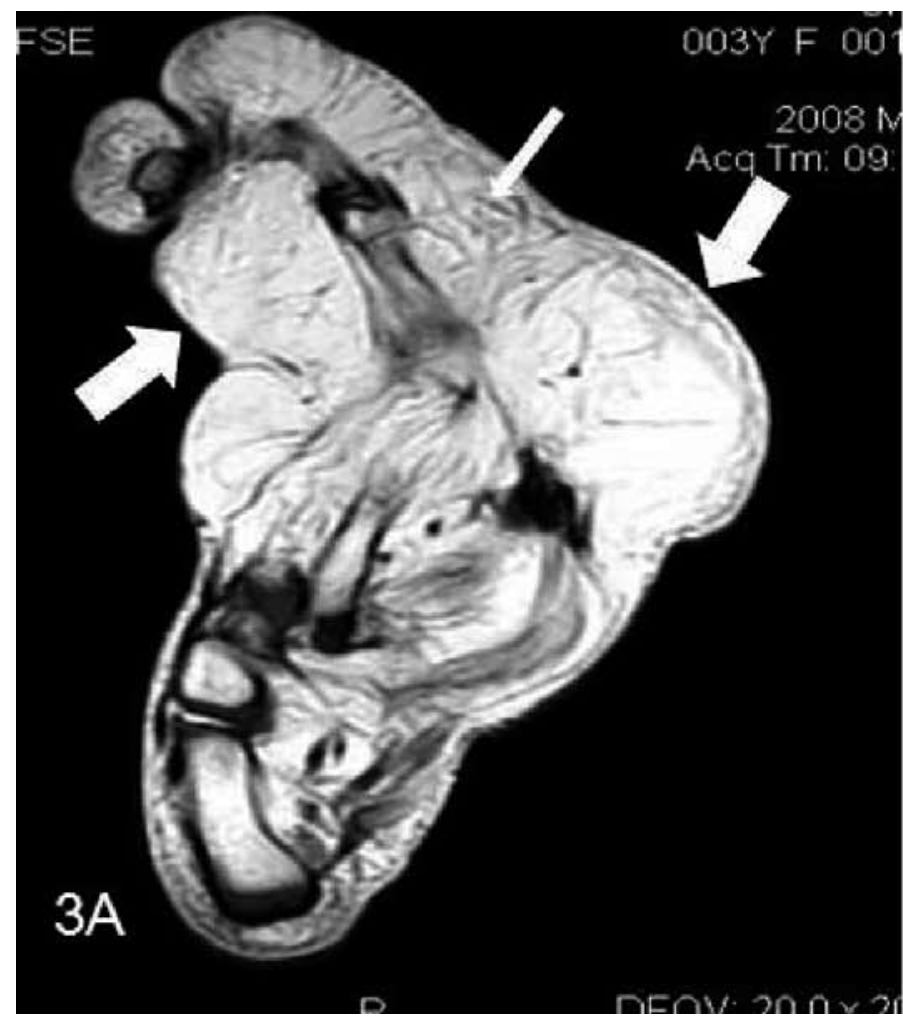

Fig. 3A. T1W sagittal images of the right foot showing local gigantism owing to gross hypertrophy of fat (thick white arrows) and soft tissue in a striated pattern ( thin white arrow).
MRI of the hand showed focal hypertrophy of the thumb and index finger (distribution of median nerve) owing to enlarged soft tissue showing a striated pattern with fatty infiltration. The soft tissue displayed hyperintense signals on T2- and T1-weighted imaging (WI) with a few linear hypointense areas alternating with hyperintense areas on both T1- and T2- weighted images (Fig. 2). On fat-suppressed shorttau inversion recovery (STIR) images, hypointensity was seen within the lesion suggestive of fatty componentsforming the bulk of the soft-tissue swelling. Numerous bony excrescences resembling small osteophytes and osteochondromas were seen in involved phalanges. No definite flow voids were seen within the lesion.

\section{Case 3}

A 3-year-old boy was referred to us from the Department of Plastic Surgery, with a history of a huge painless swelling involving his right foot since birth. Physical findings were consistent with the above two cases. MRI showed evidence of local gigantism in the foot, involving the 1st, 2nd and 3rd digits owing to gross hypertrophy of fat and soft tissue in a striated pattern. Hyperextension of the great toe was noted, with hypertrophy of metatarsals and phalanges of the great toe and widening of the first web space. The underlying metatarsals appeared broadened, suggestive of macrodactyly. Varying signal patterns consistent with bony sclerosis, oedema and erosions were seen along with subluxation of bones at the ankle joint (Figs $3 \mathrm{~A}$ and $3 \mathrm{~B}$ ).

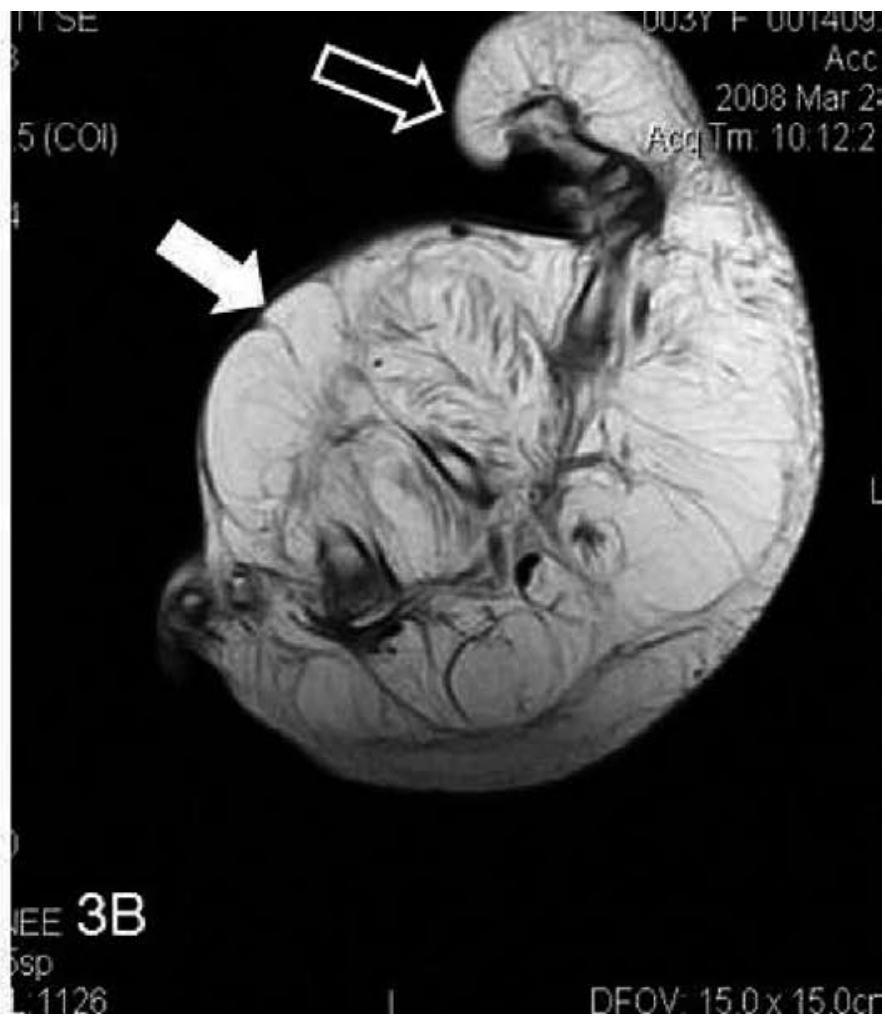

Fig. 3B. T1W axial image of the right foot showing gross hypertrophy of fat and soft tissue in a striated pattern (thick white arrow). Hyperextension of the great toe is noted, with hypertrophy of metatarsals and phalanges of the great toe and widening of the first web space (open white arrow). 


\section{Discussion}

Macrodystrophia lipomatosa was first described by Feriz in 1925 and is a rare cause of focal gigantism by deposition of fat in the soft tissue of one or more digits of the hand or foot in association with hypertrophy of the phalanges and affected limbs. ${ }^{2}$ Described as a rare congenital condition, it is classified with other phakomatoses, such as tuberous sclerosis and neurofibromatosis. ${ }^{3.5}$ The precise aetiology is unknown; however, there are many hypotheses suggesting that it is due to fibrofatty tumours impinging on nerves supplying the enlarged digit, or due to alteration of somatic cells during limb bud development. It is now believed to be a part of generalised hamartomatous disorder featuring fat in the soft tissues and even in the bowel, known as Proteus syndrome. ${ }^{67}$

The characteristic feature of the condition is that the anatomical location of the abnormality corresponds closely with the zone of innervation by the sclerotome.' Involvement of the 2 nd and 3rd digits of the hands and feet is common, corresponding to the median and the medial plantar nerve supply in the upper and lower limbs respectively; but 5 th digit involvement is rare. ${ }^{1 . s 9}$ Major clinical features are gigantism of a digit, hands, feet or the entire extremity since birth. Associated features are lipomas over other parts of the body or numerous bony excrescences resembling small osteochondroma or osteophytes (seen in Case 2) as well as associated secondary osteoarthritic changes and carpal tunnel syndrome in long-standing cases.' The patient usually seeks surgical correction for cosmetic reasons; however, mechanical problems caused by secondary osteoarthritis in early adulthood could also be the presenting problem.

Blackskin et al. were the first to describe the MR findings of this entity. ${ }^{10} \mathrm{MRI}$, owing to its inherently excellent soft-tissue contrast, is of great value in demonstrating excess fibrofatty tissue, which has signal characteristics similar to subcutaneous fat, showing high signal intensity on T1-weighted, intermediate signal on T2-weighted, and low signal on fat-suppressed. This fat is not encapsulated, as opposed to a lipoma. MRI can also well demonstrate fatty infiltration of muscles, bony overgrowth and cortical thickening. Linear hypointense bands within the excess fatty tissue, representing fibrous strands, are well visualised. Similar MR findings were demonstrated in all our 3 patients, with the fat appearing to be unencapsulated. MRI may also demonstrate fibrolipomatous hamartoma of the nerve of the affected region in some cases. ${ }^{79}$ We did not find this in our patient.

Radiologists, using different imaging modalities, play a very important role in diagnosing this condition. The diagnosis could be suspected on plain radiograph but MRI is the better diagnostic imaging modality as it can rule out other differential diagnoses such as neurofibromatosis, haemangiomatosis, lymphangiomatosis, Proteus syndrome and fibrolipomatous hamartoma. Neurofibromas are seen in close relation to nerves and show marked hyperintensity on T2-WI.
The absence of cutaneous lesions, a negative family history and sclerotomal involvement with characteristic MRI findings establish a diagnosis of MDL." Lymphangiomas have a signal intensity similar to or slightly less than muscle on T1-WI, and greater than fat on T2-WI, owing to the ectatic channels containing clear fluid. Visible septations are also seen on MRI. ${ }^{12}$ MRI imaging in haemangiomatosis demonstrates vascular channels and fibrous strands, with a palpable bruit clinically. Osseous overgrowth is not a feature of both these entities. Proteus syndrome may mimic MDL owing to its asymmetric overgrowth of bones and adipose tissue; however, associated features of macrocrania, intracranial abnormalities, cutaneous thickening, pigmented naevi, lung cysts and intra-abdominal lipomas distinguish it from MDL. Some consider MDL to be a localised form of Proteus syndrome." Fibrolipomatous hamartomas (FLH) of nerves is a rare tumour-like condition in which there is fatty deposition within nerve sheaths. The median nerve of the hand is the most commonly involved. When this disorder is associated with macrodactyly, it presents as a diagnostic dilemma with MDL. In as much as $30.7 \%$ of cases of MDL, FLH is an associated finding. ${ }^{13}$ MRI of MDL characteristically demonstrates fatty deposition not only in nerve sheaths but also in soft tissues, bone marrow and periosteum. Hence, MRI plays an important diagnostic role because of the practical importance of differentiation of these conditions as they differ in their course, prognosis, complications and treatment.

1. Sone M, Ehara S, Tamakawa Y, Nishida J, Honjoh S. Macrodystrophia lipomatosa: CT and MR findings. Radiat Med 2000; 18: 129-132.

2. Feriz H. Makrodystrophia lipomatosa progressiva. Virchows Arch 1925; 260: 308-368.

3. Murray RO, Jackobson HG, Stoker DJ. The Radiology of Skeletal Disorders: Exercises in Diagnosis, 3rd ed. New York: Churchill Livingstone, 1990: 1384.

4. Broadwater BK, Major NM, Goldner RD, LayField LJ. Macrodystrophia Lipomatosa with associated fibrolipomatous hamartoma of the median nerve. Pediatric Surg 2000; 16: 216-285.

5. Boren WL, Henry RE Jr, Wintch K. MR diagnosis of fibrolipomatous hamartoma of nerve: association with nerve territory oriented macrodactyly [macrodystrophia lipomatosa]. Skeletal Radiol 1995; 24: 296297.

6. Gupta SK, Sharma OP, Sharma SV, Sood B, Gupta S. Macrodystrophia lipomatosa: Radiographic observations. Br J Radiol 1992; 65: 769-773.

7. Balakrishna BV, Prasad S. Macrodystrophia lipomatosa - a rare congenital disorder. Indian J Radiol Imaging 2005; 15: 349-352.

8. Hafeez S, Syed M, Syed A. Macrodystrophia lipomatosa -letter to the editor. JK-Practitioner 2006; 13: 41-42.

9. Dayal J, Tyagi AR, Jain P, Ranjan R, Sharma A. Images: Macrodystrophia lipomatosa. Indian J Radiol Imaging 2001; 11: 139-141.

10. Blackskin M, Barnes FJ, Lyons MM: MR diagnosis of macrodystrophia lipomatosa. Am J Roentgenol 1992; 158: 1295-1297.

11. Singla V, Virma V, Tuli P, Khandelwal N. Case Report : Macrodystrophia lipomatosa - illustration of two cases. Indian J Radiol Imaging 2008; 18: 298-301.

12. Seigel MJ, Glazer HS, St. Amour TE, Rosenthal DD. Lymphangiomas in children: MR imaging. Pediatric Radiol 1989; 170: 467-470

13. Cavallaro MC, Taylor JA, Gorman JD, Haghighi P, Resnick D. Imaging findings in a patient with fibrolipomatous hamartoma of the median nerve. Am J Roentgenol 1993; 161: 837-838. 